\title{
MONITORAMENTO DO CONSUMO DE ENERGIA ELÉTRICA EM TEMPO REAL
}

\section{MONITORING OF ELECTRIC ENERGY CONSUMPTION IN}

REAL TIME

Fabiano Bergamin Junior

Cristiano Minotti

\section{RESUMO}

O trabalho realizado é um protótipo, de monitoramento do consumo de energia elétrica de uma residência, onde utilizam os conceitos de internet of things, permitindo que o dispositivo possa se conectar a internet, através da tecnologia wi-fi e disponibilizar os dados do consumo em quilowatt hora, em uma plataforma de cloud, onde possa ser acessado em tempo real por meio smartphone.

Palavras-chave: Medidor de consumo de Energia Elétrica. Casa Inteligente. ESP32. IoT.

\begin{abstract}
The work carried out is a prototype, monitoring the electricity consumption of a home, where it uses the concepts of internet of things, allowing the device to connect to the internet, using wireless technology and making consumption data available in kilowatt hours. , on a cloud platform, where it can be accessed in real time through the smartphone.
\end{abstract}

Keywords: Electricity. ESP32. loT. Smart Home 


\section{TOUNARA \\ UNIVERSIDADE DE ARARAQUARA}

\section{INTRODUÇÃO}

Com o surgimento de novos tecnologias em eletrônica (hardwares) e o consequente aumento da capacidade de processamento dos dispositivos eletrônicos embarcados, tornou-se possível criar dispositivos que se comunicam com internet, conhecidos por Internet das Coisas ou loT (do inglês, Internet of Things). O conceito de loT, nasceu no início dos anos 2000, onde Kevin Ashton estabeleceu a base que seria a internet das coisas em um laboratório do MIT, ao realizar pesquisa sobre RFID (Radio Frequency Identification), onde objetos eram equipados com identificadores e conectividade sem fio, onde poderiam se comunicar e serem gerenciados pelos computadores (CISCO, 2013).

Segundo as projeções realizadas sobre a conectividade dos dispositivos com a internet até 2023, a M2M (do inglês, Machine To Machine) que é a utilizada pelo loT, representará cerca de 50\% da conexão global, representando 14,7 bilhões de dispositivos conectados, como ilustra a Figura 1 (CISCO, 2020). Com as projeções de crescimento de conectividade dos dispositivos embarcados loT e hardwares acessíveis, torna-se mais simples o desenvolvimento de soluções como o de um medidor de energia inteligente (do inglês, Smart Meter), que realiza medições do consumo de energia elétrica em tempo real de uma residência.

Figura 1- Crescimento global de dispositivos e conexões, medidos em CAGR ( Compound anual groth rate) ou taxa de crescimento anual composto.

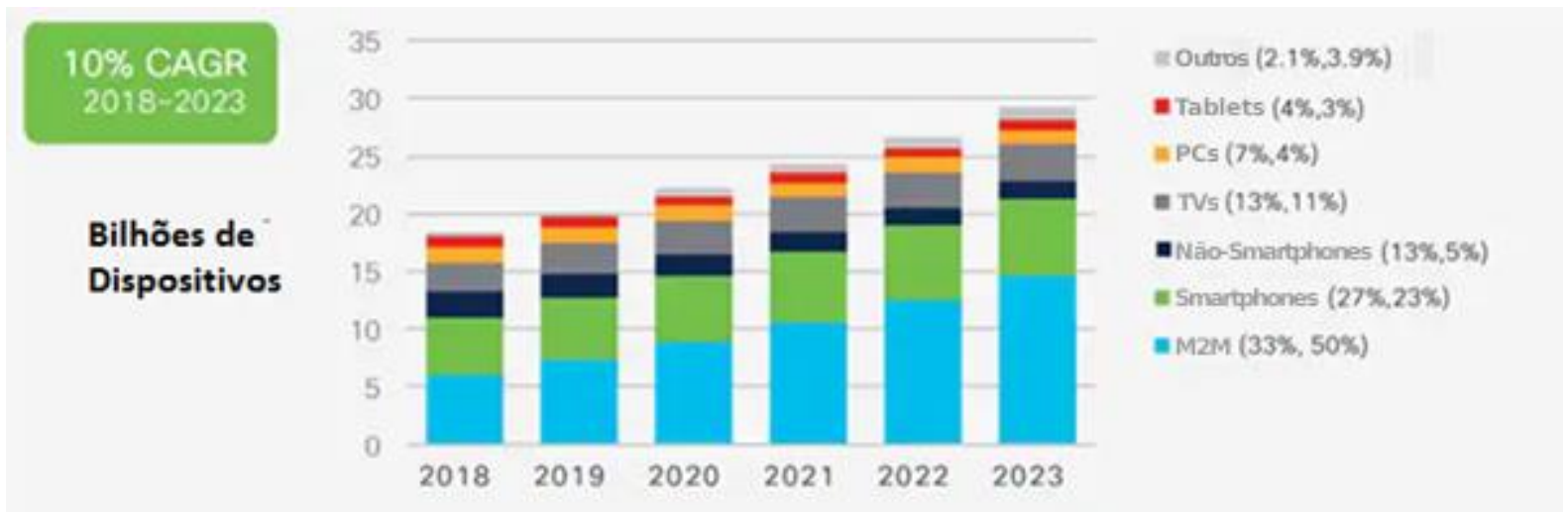

Fonte: adaptado (CISCO, 2020)

De acordo com o Balanço Energético Nacional de 2020, o consumo nacional de eletricidade de 2019 foi 545,6 TW.h (TeraWatts * Hora), tendo crescimento de 1,3\% comparado o ano anterior, onde consumo residencial, foi uns dos serviços que mais contribuiu para o crescimento do consumo nacional, com 4,8 TW.h crescimento de mais 3,5\% (EPE, 2020).

Com a pandemia devido ao novo coronavírus (COVID-19), muitas pessoas adotaram o regime de teletrabalho, onde passaram a ficar mais tempo em suas resistências e, portanto, aumentando o consumo de energia elétrica. $O$ consumo de eletricidade por uma residência é fornecido pela concessionaria com um ciclo de tarifação de um mês (28 a 30 dias) como mostra a fatura ilustrada na Figura 2 onde o usuário pode acompanhar os seus consumos mensais. 
Figura 2- Exemplo de fatura de energia elétrica fornecida pela concessionaria de energia

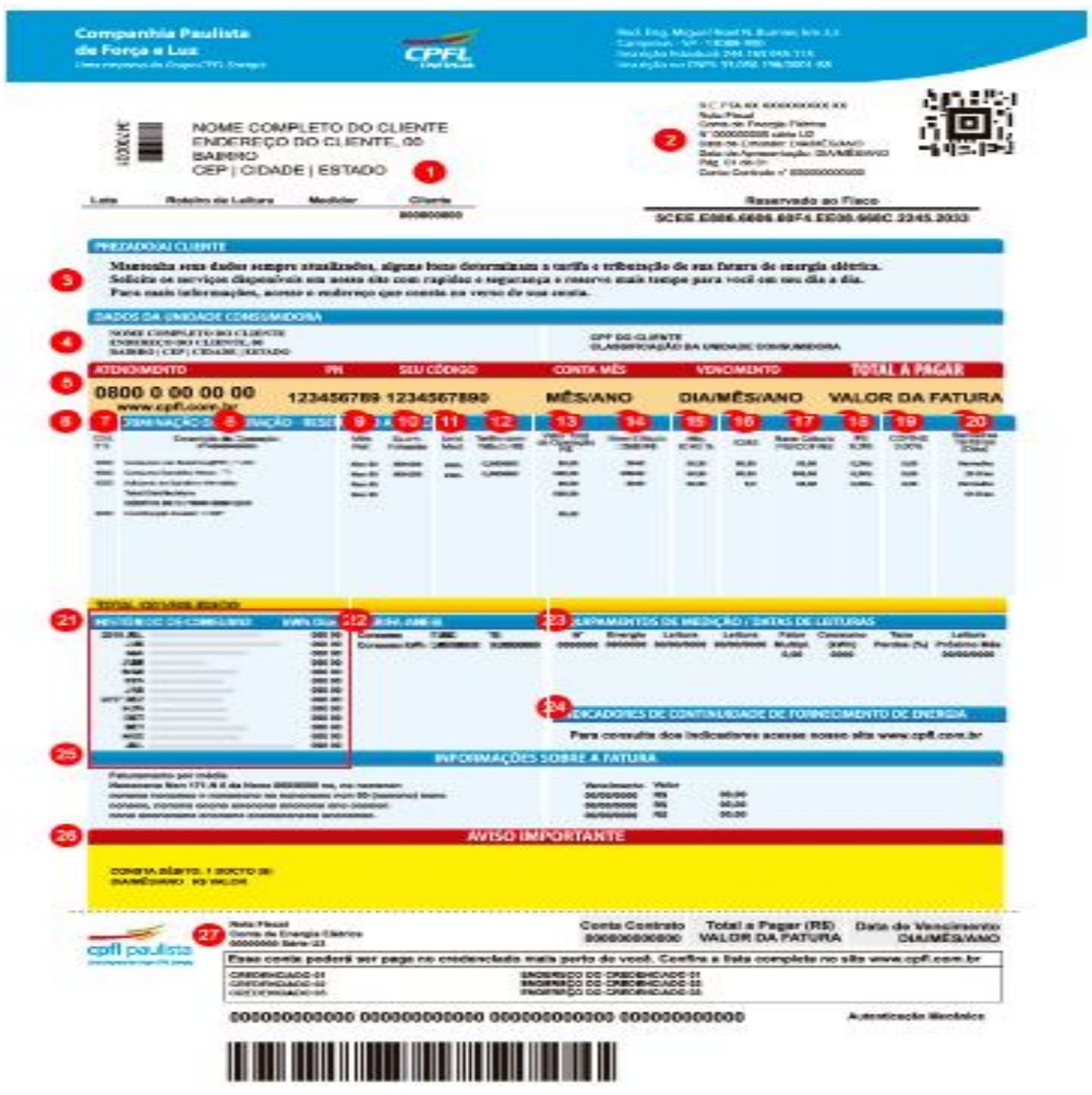

Fonte: (CPFL, 2017)

Observando os dados informados na fatura apontada na Figura 2, nota-se que há uma carência de informação sobre o consumo diário de energia elétrica, visto que os dados disponibilizados ao usuário é valor do consumo total durante o ciclo de tarifação (mensal). Com a ausência da informação apresentado na fatura do consumo de energia elétrica diário, propõe-se neste trabalho desenvolver um protótipo, que realiza medições diárias do consumo de eletricidade, disponibilizando os dados de medição para ser consultados em tempo real pelo usuário, de modo que este possa perceber os 


\section{LOUNIARA \\ UNIVERSIDADE DE ARARAQUARA}

maiores consumidores de energia me sua instalação em naquele instante. Embasado nesse contexto, este trabalho tem o objetivo de propor um medidor de energia elétrica com o conceito de internet das coisas (IoT), onde o usuário poderá acessar os dados de consumo elétrico por meio do seu smartphone.

\section{FUNDAMENTAÇÃO TEÓRICA}

Nesta sessão, são apresentadas as informações relacionadas com as tecnologias que foram utilizadas para o desenvolvimento só medidor de energia elétrica.

\subsection{Microcontrolador}

O microcontrolador é circuito integrado que é possibilita reunir num invólucro único ou componente um circuito completo que exerça determinada função, onde é possível usá-lo em projetos de automação, equipamentos de mediações e precisão, computadores de bordo entre outras aplicações de controle, onde há a necessidade da interação humano computado. Esses exemplos de aplicações são possíveis devido à composição de um microcontrolador, que contém um microprocessador ou CPU (do inglês, Central Processing Unit), que realiza operações lógicas e aritméticas com sinais digitais, além de conter uma memória de programa responsável por armazenar as instruções ou software desenvolvido para uma determinada aplicação. Possui também uma memória de dados responsável por ler as informações dos periféricos (sensores) conectados as portas I/Os (do inglês, Input And Output) (BRAGA, 2021).

\subsubsection{Esp32}

O ESP32 é um SOC (system on a chip) é um módulo eletrônico integrado que oferece conectividade (Wi-Fi e Bluetooth) e possui com poder computacional (CPU + memórias) constituído pelo microprocessador Xtensa LX6 dual core, com frequência de operação de 160 ou $240 \mathrm{MHz}$, podendo realizar cerca de 600 MIPS (do inglês, Millions of Instructions per Second) (BERTOLETI, 2019). 
Figura 3- Placa ESP32

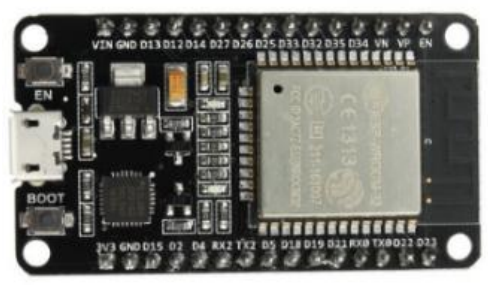

Fonte: (FLIPFLOP, 2021)

O ESP32 é uma poderosa plataforma para uso em loT, pois apresenta periféricos de comunicação (I2C, SPI e UART), pinos analógicos e digitais e RTC (do inglês, Real Time Clock). Além disso, apresenta um ambiente desenvolvimento simples, que utiliza a linguagem $\mathrm{C} / \mathrm{C}_{++}$com o Arduino IDE (BERTOLETI, 2019).

\subsection{Sensor de corrente (SCT-013-00)}

O modelo SCT-013-00 é um sensor utilizado para realizar medições de corrente alternada de forma não invasiva em condutores elétricos, que operam com tensão de 127 a 220 Volts. Segundo a folha de dados do componente (DATASHEET, 2021) o sensor permite realizar as leituras de 0 a 100 Ampères em seu enrolamento primário, que apresentará uma corrente proporcional no enrolamento secundário (bobina), que varia de 0 a $50 \mathrm{~mA}$, ou seja, trata-se de um transformador de corrente (TC). O sensor SCT-013-00 é apresentado na Figura 4.

\section{Figura 4- Sensor de corrente (SCT-013-00)}

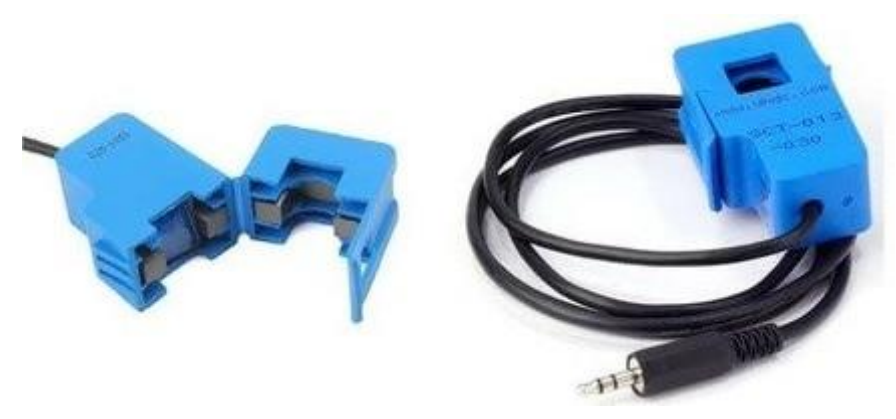

Fonte: (HOW2WLETRONICS, 2021)

\subsubsection{Circuito Eletrônico Do Sensor De Corrente.}

Segundo a folha de dados do sensor de corrente escolhido (SCT-013-00) (DATASHEET, 2021), o componente apresenta variações de medição de saída numa faixa de 0 a $50 \mathrm{~mA}$ proporcional à corrente medida Ip, sendo recomendado a aplicação de um resistor de carga paralelo a saída do sensor, como ilustra a Figura 5, permitindo que o sistema embarcado possa realizar as leituras do sinal do sensor. 


\section{TOUNIARA \\ UNIVERSIDADE DE ARARAQUARA}

Figura 5- Sensor de corrente (SCT-013-00)

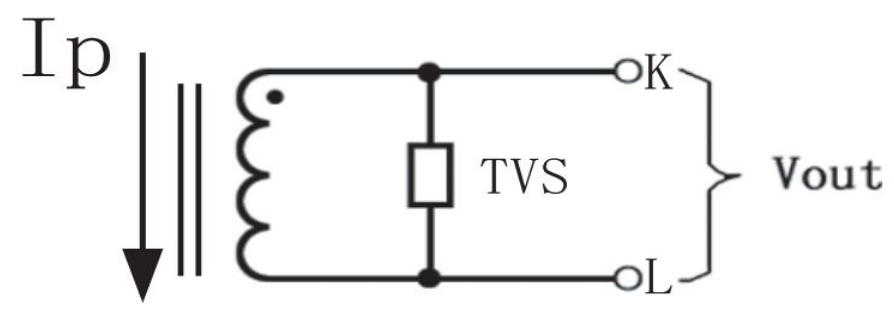

Fonte: (DATASHEET, 2021)

\subsubsection{Cálculo do Resistor de Carga}

Segundo a folha de dados do sensor de corrente escolhido (DATASHEET, 2021), o sensor SCT-013-00 é calibrado para medir a corrente máxima alternada de até 100 Amperes RMS (do inglês, Root Mean Square) ou conhecido com valor eficaz. Para o cálculo do resistor de carga ou de Burden, primeiramente deve-se que realizar a conversão da corrente eficaz para a corrente de pico primaria $\left(I_{p 1}\right)$ como ilustra a Equação (1.1).

$$
I_{p 1}=I_{r m s} \cdot \sqrt{2}=141,4 A
$$

Com a corrente de pico primaria calculada, pode-se calcular a corrente de pico secundário $\left(I_{p 2}\right)$ a que percorrerá o resistor de carga. Para esse cálculo será utilizado a equação, que permite calcular a relação de transformação do transformador, considerando o enrolamento primário e secundário igual a $N_{1}$ e $N_{2}$ respectivamente 1 e 2000 voltas, (aponta o manual do componente em sua referência bibliográfica).

$$
\frac{N_{1}}{N_{2}}=\frac{I_{p 2}}{I_{p 1}}
$$

Com a equação 1.2 e os com valores de $N_{1}, N_{2}$ e $I_{p 1}$ pode-se então isolar a equação em função de $I_{p 2}$ calcular a correte do enrolamento secundário, conforme a equação 1.3.

$$
I_{p 2}=\frac{141,4 A}{2000}=70,7 m A
$$

Para melhora a resolução de medição do ESP32, fazendo que a corrente fique na faixa de operação de tensão do ESP32, deve se utilizar a metade da tensão máxima do ESP32, que é de 1.65 $\mathrm{V}$, conforme a Equação 1.4. Portanto:

$$
R_{\text {burden }}=\frac{1.65}{0,0707}=22 \Omega
$$


UNIVERSIDADE DE ARARAQUARA

Com o resistor de carga dimensionado $\left(R_{\text {burden }}\right)$, este projeto tem a necessidade de utilizar um circuito de condicionamento ilustrado na Figura 6, conhecido como divisor de tensão, que permite que o ESP32 possa ler o sinal de corrente em uma faixa de tensão de 1,65 a 3.3 Volts, eliminado a tensão do ciclo negativo e filtrando o sinal do semiciclo positivo, através do capacitor eletrolítico $C_{1}$, que será lido pelo conversor AD (Analógico/Digital) do ESP32.

Figura 6- Circuito de condicionamento do resistor de carga

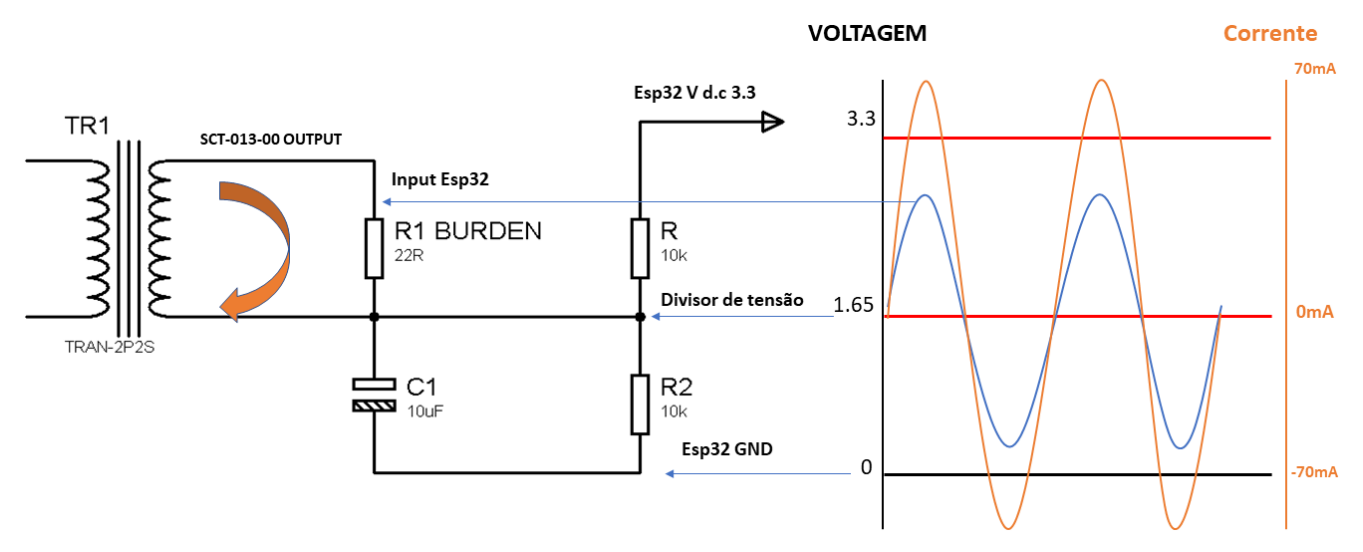

Fonte: PRÓPRIA (2021)

Com o levantamento dos requisitos necessários para leitura do sinal do sensor SCT-013-000, foi montado o circuito eletrônico de condicionamento, onde basicamente consiste em um divisor de tensão composto por dois resistores de $10 \mathrm{~K} \Omega$ e o circuito de desacoplamento, constituído pelo capacitor eletrolítico de $10 \mu \mathrm{F}$, ilustrado na Figura 7.

Figura 7- Circuito e medição da correte elétrica

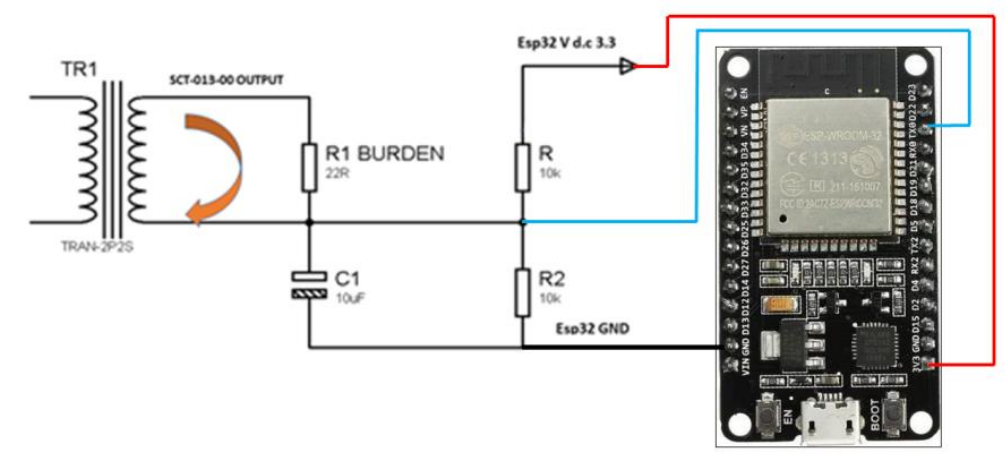

Fonte: PRÓPRIA (2021) 


\subsection{Sistema operacional Android}

O Android é o sistema operacional mobile mais utilizado no mundo utilizado por mais de dois bilhões de dispositivos ativos, presentes em smartphones, tablets, TVs, relógios entre outros (GLAUBER, 2019).

O Android é um sistema operacional open-source: onde seu código fonte, pode ser baixado, modificado e acessado por: qualquer indivíduo sob a licença Apache, onde permite que qualquer fabricante de dispositivos móveis possa utilizá-lo e modificá-lo através das AOSP (Android Open Soure Project). Contudo, para que o aparelho saia da fábrica com o Android, é necessário que o dispositivo tenha os aplicativo do Google (Gmail, Maps, Google Play e You Tube) (GLAUBER, 2019).

\subsubsection{Blynk}

O Blynk é uma plataforma de loT desenvolvida para os sistemas iOS e Android, onde permite que dispositivos embarcados, possam se conectar a ela de forma simples, por meio da internet. Assim, o usuário pode criar uma dashboard (interface gráfica), sem complexidade, apenas as arrastando e soltando os seus widgets (componentes de tela, prontos para ser utilizados), como pode ser visto na Figura 8.

Figura 8- Interface do Aplicativo Blynk com os widgets

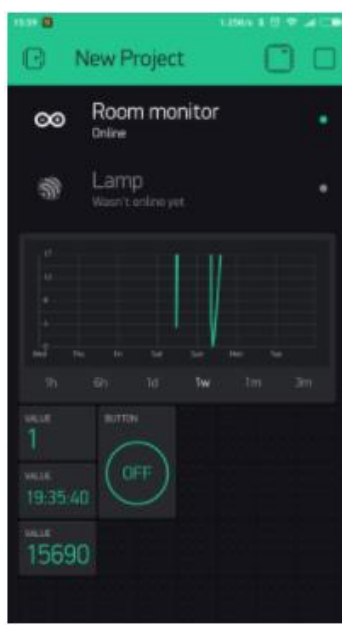

Fonte (Blynk, 2021) 


\section{MATERIAIS E MÉTODOS}

Primeiramente, o desenvolvimento do protótipo iniciou-se, pela montagem do circuito eletrônico, onde consiste em circuito de polarização responsável por excursionar o sinal medido pelo sensor de corrente. Em seguida iniciou-se o desenvolvimento do sistema embarcado responsável pelas leituras do sensor e enviar os dados para o aplicativo Blynk, onde os dados poderão ser visualizados pelo dashboard, permitindo ao usuário ver a amostragem dos dados durante o dia e a o consumo no exato momento que estiver com o aplicativo.

\section{RESULTADOS E DISCUSSÃO}

Para a validação do medidor de energia elétrica, foi construído uma pequena estrutura em madeira, constituído por dois interruptores, para simular um circuito elétrico com duas lâmpadas incandescente halógenas, para simular cargas resistivas, instaladas em paralelo onde os elementos que constituem o circuito, encontram em paralelo com a fonte de energia (CAVALIN e CERVELIN, 2009), como ilustra a Figura 9.

\section{Figura 9- Circuito elétrico de medição do consumo de energia elétrica}
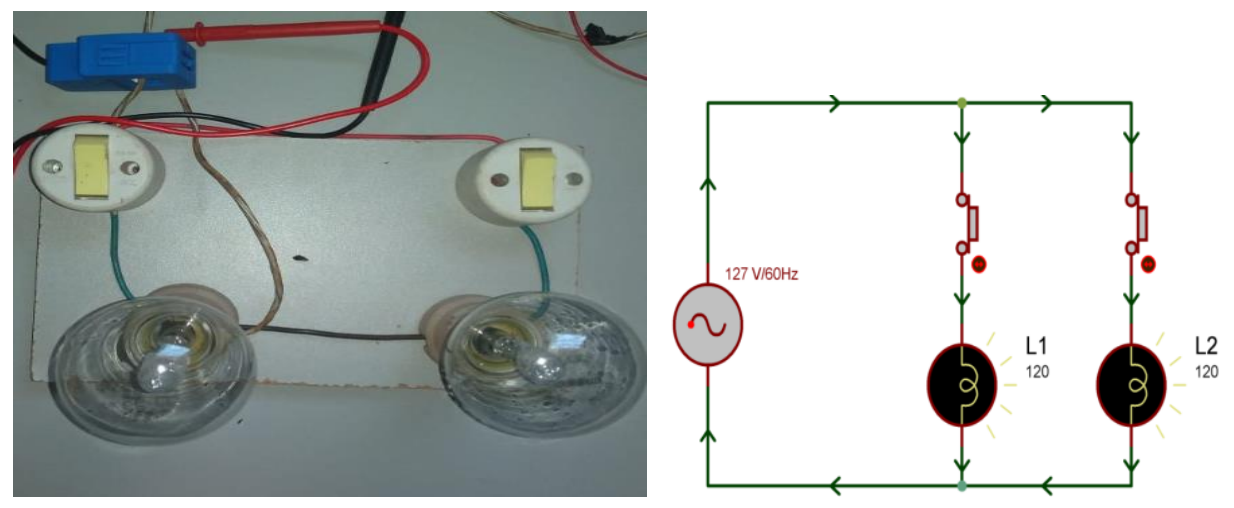

Fonte: PRÓPRIA (2021)

Como pode ser ver na Figura-5, o sensor SCT-013-00, está realizado a medição do consumo da corrente elétrica das lâmpadas, que simulam cargas resistiva em uma residência. $O$ sensor de corrente não invasivo em via os sinais das correntes, para o circuito de carga, que está conectado ao sistema embarcado (ESP32), que por sua vez processa os sinais, através de um conversor analógico digital, como poder ser visto na Figura 10. 
UNIVERSIDADE DE ARARAQUARA

Figura 10- Circuito eletrônico de carga e conectado com o sistema embarcado.

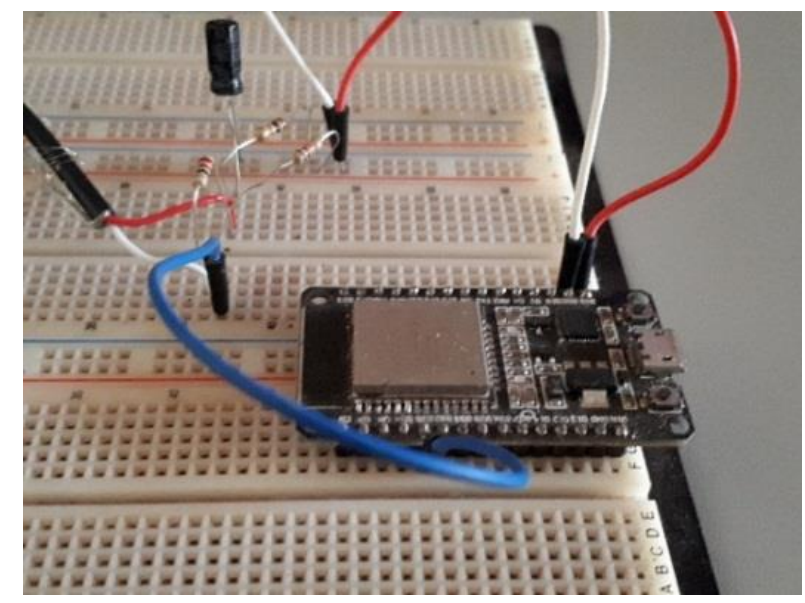

Fonte: PRÓPRIA (2021)

Para os testes de medição, foram considerados dois cenários para medir a corrente elétrica, o primeiro: utilizou duas lapadas incandescente de 120 watts, e o segundo cenário utilizou lâmpadas incandescentes de 70 e 120 watts de potência. Para a realização do cálculo do custo de medição, utilizou-se da tarifa de cobrança, estipulada pela ANEEL(Agência Nacional de Energia Elétrica), que se encontra na futura da concessionária de energia, que no caso foi utilizado o TE (Tarifa de Consumo de Energia) e o TUDS (Tarifa de Uso do Sistema de Distribuição), onde o consumo está sendo calculado, a cada segundo de leitura (taxa de amostragem), de forma acumulativa, ou seja, assim que o sistema embarcado é inicializado o valor de consumo é acumulativo.

Como dito no parágrafo anterior, o primeiro cenário se encontra o aferimento, com duas lâmpadas de 120 watts de potência, onde possa ser visualizado em tempo real, o consumo da potência, custo e corrente elétrica, através do smartphone, como ilustra a Figura 11.

Figura 11- Cenário de teste de 240 watts de potência.
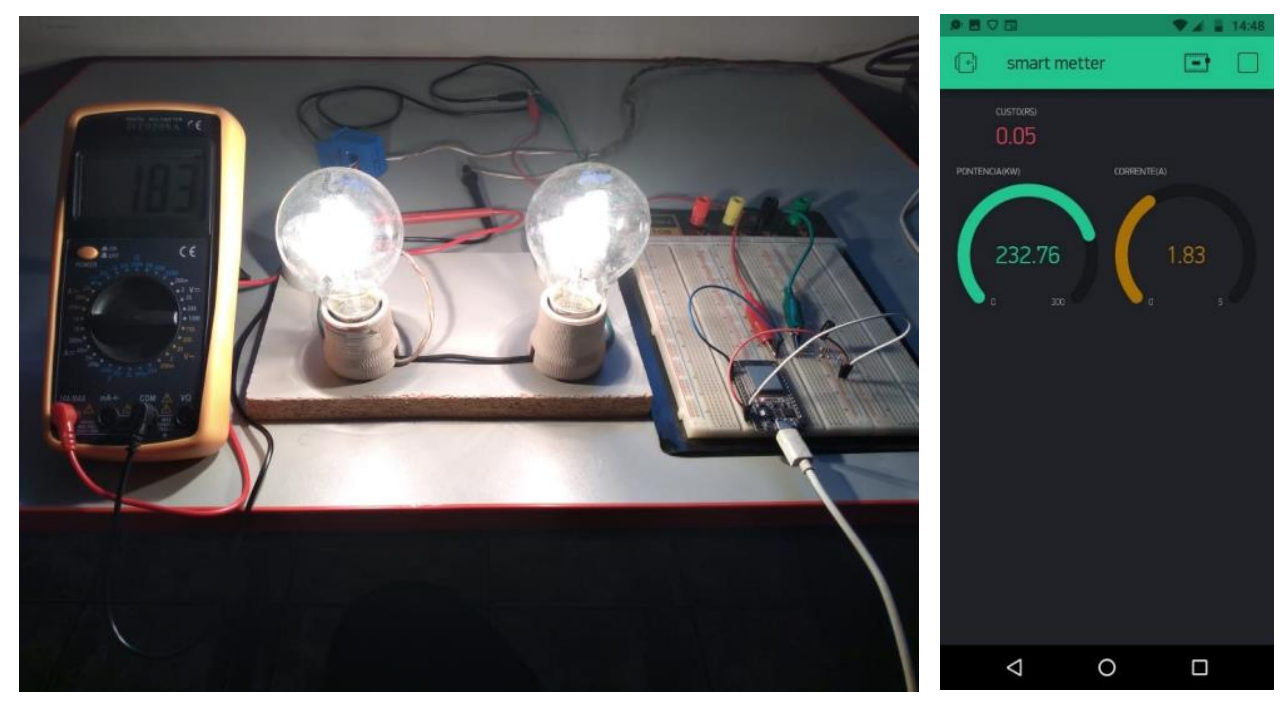

Fonte: PRÓPRIA (2021)

O cenário de teste de 240 watts de potência, apresentou medições de 1,83 amperes de corrente, esse valor foi mesurando pelo sensor não invasivo e aferido e comparado, com um instrumento de medição (multímetro), para verificar a acurácia do sensor SCT-013-00. Em seguido o 
UNIVERSIDADE DE ARARAQUARA

teste com o cenário de 170 watts de potência, foi realizado para verificar a acurácia do sensor não invasivo, como visto na Figura 12.

Figura 12- Cenário de teste de 170 watts de potência.
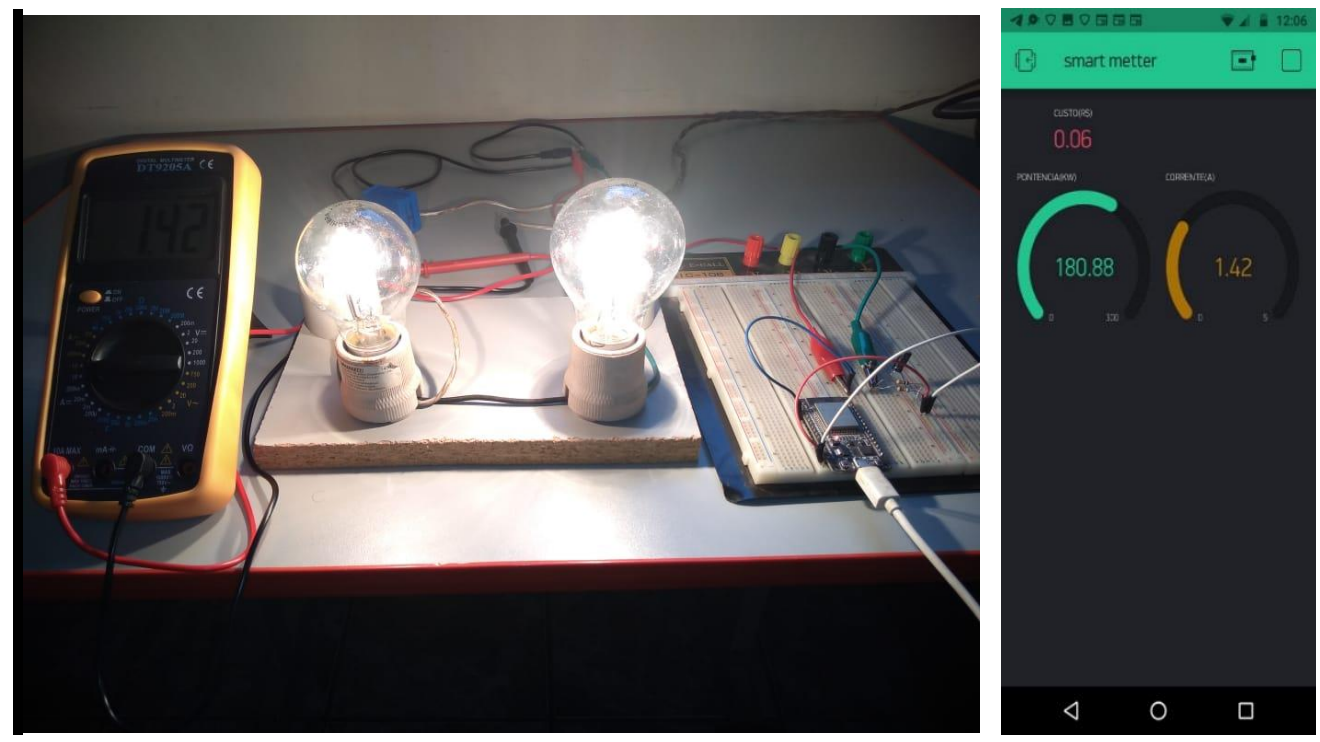

Fonte: PRÓPRIA (2021)

O cenário de teste de 170 watts de potência, apresentou o consumo de 1,42 amperes, esse valor de leitura, foi apresentado pelo sensor de corrente e pelo instrumento de medição, que verificou a acurácia e exatidão das leituras realizadas.

\section{CONSIDERAÇÕES FINAIS}

Este artigo apresentou o desenvolvimento de um projeto de monitoramento do consumo de energia elétrica em tempo real, com a capacidade de informar o responsável da residência, de forma visual, através de um dashboard, criado com componentes visuais presentes no aplicativo Blynk, onde representam grandezas elétricas como: corrente e potência, além do custo monetário. Durante o desenvolvimento do software do sistema embarcado, o conversor analógico digital do ESP32, apresentou instabilidade para a leitura do sensor SCT-013-00, devido uma alta flutuação de sinal, ouve a necessidade de corrigir essa instabilidade, por meio de software, onde foi realizado uma amostragem de cem leituras e calculado a média ponderada. O teste de conexão wi-fi com o ESP32 e o aplicativo Blynk, apresentou: ótimo atualização de resposta dos dados, ao usuário. Com esses resultados o protótipo se mostrou ser uma alternativa viável para realizar o monitoramento da energia elétrica residencial, que apresentam cargas resistivas, porém nos cenários, onde há a utilização de cargas capacitivas e indutivas o sistema, irá apresentar erro de medição, devido estar aferindo a corrente aparente invés da eficaz. 
Referências

BERTOLETI, P. PROJETO COM ESP32 E LORA. SÃO PAULO: [s.n.], 2019.

BLYNK. https: //blynk.io/, 23 maio 2021. Disponivel em: <http://docs.blynk.cc/\#intro-how-blynkworks>.

BRAGA, N., 27 fev. 2021. Disponivel em: <https://www.newtoncbraga.com.br/index.php/eletronica/52artigos-diversos/13263-o-basico-sobre-os-microcontroladoresparte-1-mic139>.

CAVALIN, G.; CERVELIN, S. INSTALACOÕES ELÉTRICAS PREDIAIS. 19. ed. SÃO PAULO: ÉRICA, 2009.

CISCO, 2013. Disponivel em:

$<$ https://www.cisco.com/c/dam/global/pt_br/assets/brand/iot/iot/pdfs/lopez_research_an_introduction_t o_iot_102413_final_portuguese.pdf>.

CISCO, 2020. Disponivel em: <https://www.cisco.com/c/en/us/solutions/collateral/executiveperspectives/annual-internet-report/white-paper-c11-741490.html>.

CPFL, 30 jun. 2017. Disponivel em: <https://www.cpfl.com.br/releases/Paginas/cpfl-energia-lancanova-conta-de-energia-eletrica-para-7-9-milhoes-de-clientes-em-sp-e-no-rs.aspx>. Acesso em: 27 fev. 2021.

DATASHEET, 2021. Disponivel em:

<https://img.filipeflop.com/files/download/Datasheet_SCT013.pdf>. Acesso em: 27 fev. 2021.

EPE, 2020. Disponivel em: <https://www.epe.gov.br/pt/publicacoes-dados-

abertos/publicacoes/balanco-energetico-nacional-2020>. Acesso em: 20 fev. 2021.

FLIPFLOP, 2021. Disponivel em: <https://www.filipeflop.com/produto/modulo-wifi-esp32-bluetooth/>. Acesso em: 27 fev. 2021.

GLAUBER, N. Dominando o Android com Kotlin. SÃO PAULO: NOVATEC, 2019.

HOW2WLETRONICS, 10 mar. 2021. Disponivel em: <https://how2electronics.com/iot-basedelectricity-energy-meter-using-esp32-blynk/>. Acesso em: 14 mar. 2021. 